\title{
New results on SIDIS SSA from Jefferson Lab
}

\author{
H. Avakian, P. Bosted, V. Burkert, L. Elouadrhiri for the CLAS Collaboration \\ Jefferson Lab, Newport News, VA 23606, USA
}

\begin{abstract}
We present studies of single-spin and double-spin asymmetries in semi-inclusive electroproduction of pions using the CEBAF $6 \mathrm{GeV}$ polarized electron beam. Kinematic dependences of single and double spin asymmetries have been measured in a wide kinematic range at CLAS with a polarized $\mathrm{NH}_{3}$ target. Significant target-spin $\sin 2 \phi$ and $\sin \phi$ asymmetries have been observed. The hypothesis of factorization has been tested with $z$-dependence of the double spin asymmetry.
\end{abstract}

Single-Spin Asymmetries (SSAs) in azimuthal distributions of final state particles in semi-inclusive deep inelastic scattering play a crucial role in the study of transverse momentum distributions of quarks in the nucleon and provide access to the orbital angular momentum of quarks. Large SSAs, observed for decades in hadronic reactions have been among the most difficult phenomena to understand from first principles in QCD. Recently, significant SSAs were reported in semi-inclusive DIS (SIDIS) by the HERMES collaboration at HERA [1, 2] for longitudinally and transversely polarized targets, and by the CLAS collaboration with a polarized beam [3].

Two fundamental mechanisms have been identified leading to SSAs in hard processes, the Sivers mechanism [4, 5, 6, 7, 8], which generates an asymmetry in the distribution of quarks due to orbital motion of partons, and the Collins mechanism [9, 10], which generates an asymmetry during the hadronization of quarks.

The HERMES Collaboration has recently measured a transverse spin asymmetry in SIDIS providing the cleanest evidence to date for the existence of a non-zero Collins function [2], which describes the fragmentation of a transversely polarized quark into pions. This finding is supported by the preliminary data from BELLE [11] indicating a non-zero Collins effect. The large target SSA in semi-inclusive pion production measured at CLAS and analyzed in terms of the Collins fragmentation [12], also indicate a significant Collins function.

For a longitudinally polarized target the only azimuthal asymmetry arising in leading order is the $\sin 2 \phi$ moment [15, 10, 13], involving the transverse momentum dependent (TMD) Collins fragmentation function $H_{1}^{\perp}[9]$ and the Mulders distribution function $h_{1 L}^{\perp}$ [14, 10], describing the transverse polarization of quarks in a longitudinally polarized proton [15, 10, 13]. The same distribution function is accessible in double polarized Drell-Yan, where it gives rise to a $\cos 2 \phi$ azimuthal moment in the cross section [16].

Single and double spin asymmetries in SIDIS have been measured using the CLAS [17] in Hall B at Jefferson Lab, a $5.7 \mathrm{GeV}$ longitudinally polarized electron beam, a longitudinally polarized proton $\left(\mathrm{NH}_{3}\right)$ target. The average beam polarization was $0.73 \pm 0.03$ and the average target polarization was $0.72 \pm 0.05$. The open acceptance of CLAS and a single electron trigger ensured event recording for a large sample of SIDIS 
$\pi^{+}, \pi^{0}$, and $\pi^{-}$events.
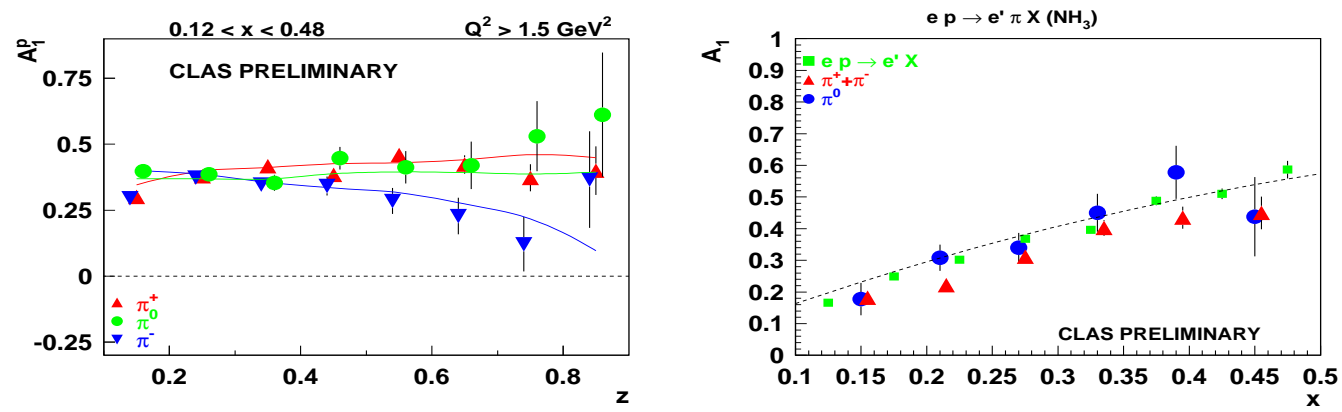

FIGURE 1. The double spin asymmetry for SIDIS $\pi^{+}, \pi^{-}$, and $\pi^{0}$ data as a function of $z$ (left) and the comparison of inclusive $A_{1}^{p}$ with the $A_{1}^{p}$ for the SIDIS sum of $\pi^{+}$and $\pi^{-}$and $A_{1}^{p}$ for SIDIS $\pi^{0}$ (right).

The validity of factorization is crucial to the interpretation of target SSAs in terms of TMDs, which is the main goal of this contribution. A factorization test comes from examining the $z$-dependence of the double spin asymmetries $\left(A_{1}^{p}\right)$ for all three pion flavors, as shown in the left panel of Fig. 1. The data cuts included $W>2 \mathrm{GeV}$ and $Q^{2}>1.1 \mathrm{GeV}^{2}$ to ensure DIS kinematics, and the average value of $x$ is approximately 0.3 . If factorization holds, the asymmetries should be approximately independent of $z$, broken by the different weights given to the polarized $u$ and $d$ quarks by the favored and unfavored fragmentation functions. We therefore expect the largest $z$-dependence for the $\pi^{-}$asymmetries. This is indeed born out by the data, which are in excellent agreement in both magnitude and $z$-dependence up to $z=0.7$ with predictions of the polarized Lund Monte Carlo using GRSV polarized PDFs as input.

The dependence of the double spin asymmetry on Bjorken $x$ for different pion flavors obtained from the CLAS data for the same kinematic range is presented in Fig. 11(right panel). The $\pi^{0}$ double spin asymmetry as well as $A_{1}^{p}$ for the sum of charged pions are consistent with the inclusive $A_{1}^{p}$ as expected in a simple partonic picture.

These studies suggest that factorization works for $W>2 \mathrm{GeV}, Q^{2}>1.1 \mathrm{GeV}^{2}$, $0.15<x<0.5$, and $0.3<z<0.7$ for a $5.7 \mathrm{GeV}$ electron energy.

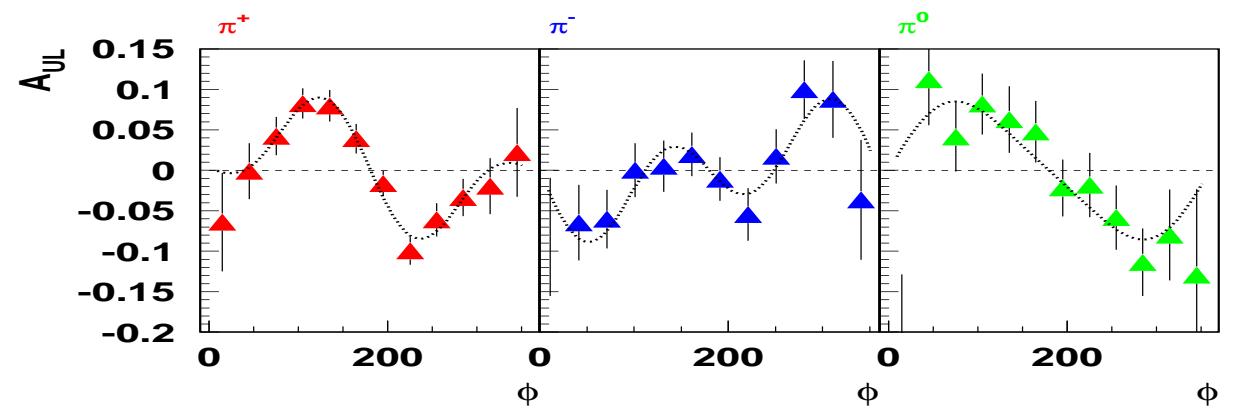

FIGURE 2. The target SSA as a function of azimuthal angle $\phi$ from data at $5.7 \mathrm{GeV}$.

The spin-dependent moments $(\sin \phi, \sin 2 \phi)$ of the semi-inclusive cross section have been extracted in a fit of the normalized-yield asymmetry 


$$
A_{U L}(\phi)=\frac{1}{P_{T}} \frac{N^{+}-N^{-}}{N^{+}+N^{-}}
$$

Here $N^{ \pm}$is the number of events for target polarizations antiparallel/parallel to the incoming beam direction and $P_{T}$ is the target polarization.

Measurements of the $\sin 2 \phi$ SSA allow the study of the Collins effect with no contamination from other mechanisms. A recent measurement of $\sin 2 \phi$ moment of $\sigma_{U L}$ by HERMES [1] is consistent with zero. A measurably large asymmetry has been predicted only at large $x(x>0.2)$, a region well-covered by JLab [18].

The data for $\pi^{+}$(Fig. 22) show a clear $\sin \phi$ and $\sin 2 \phi$ modulations from which a $\sin \phi$ moment of $0.058 \pm 0.011$ (stat) and $\sin 2 \phi$ moment of $-0.041 \pm 0.011$ (stat) have been determined.
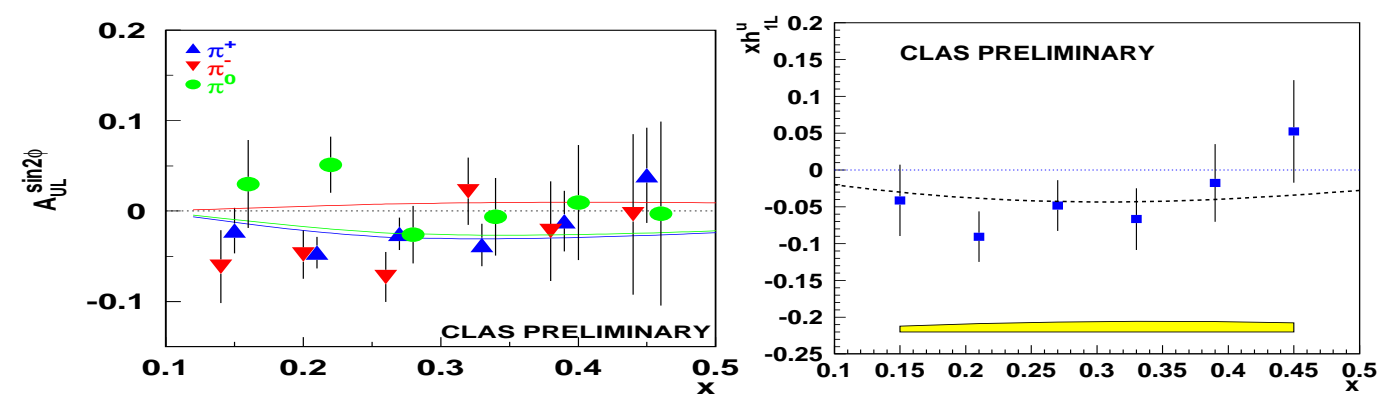

FIGURE 3. The leading twist SSA for the $\sin 2 \phi$ moment for $\pi^{+}, \pi^{0}$, and $\pi^{-}$as a function of $x$ (left plot). The $h_{1 L}^{\perp}$ from the $\pi^{+}$SSA (right). The contribution from the unfavored production is included in the systematic error band. The variation for the ratio of unfavored to favored Collins functions is from -2.5 to 0 . The curves are from [18] using the $\chi \mathrm{QSM}$ calculations of $h_{1 L}^{\perp}$.

The $x$ dependence of the SSA for $\pi^{+}$(Fig. 3) is consistent with predictions [18]. No sign of a large unfavored Collins fragmentation (large $\pi^{-}$SSA with a corresponding $\pi^{+}$SSA of opposite sign) is seen. The $\pi^{+}$SSA is dominated by the u-quarks; therefore with some assumption about the ratio of unfavored to favored Collins fragmentation functions, it can provide a first glimpse of the twist-2 TMD function $h_{1 L}^{\perp}$ (Fig. 3.) The curve is the calculation by Efremov et al. [18], using $h_{1 L}^{\perp}$ from the chiral quark soliton model evolved to $Q^{2}=1.5 \mathrm{GeV}^{2}$. The extraction, however, suffers from low statistics and has a significant systematic error from the unknown ratio of the Collins favored and unfavored fragmentation functions, the unknown ratio of $h_{1 L}^{\perp d} / h_{1 L}^{\perp u}$, as well as from background from exclusive vector mesons.

The $\sin \phi$ moment of the cross section measured with CLAS at $5.7 \mathrm{GeV}$ is in agreement with the HERMES measurement at $27.5 \mathrm{GeV}$ for a longitudinal target [1], indicating a bigger asymmetry for the higher twist contribution compared to the leading twist $\sin 2 \phi$ moment. The $P_{\perp}$ dependence of the $\sin \phi$ moment for $\pi^{+}$(see Fig. (4) is consistent with an increase with increasing $P_{\perp}$, as expected for the TMD function [19]. The $\sin \phi$ moment, for $\pi^{-}$is also plotted in Fig. 4] showing first evidence for a non-zero SSA asymmetry for $\pi^{-}$on a longitudinally polarized target.

The $\sin \phi$ moment of the SIDIS cross section itself can be an important source of independent information on the Collins fragmentation mechanism. Several other con- 
tributions to the $\sin \phi$ moment were identified recently [20, 21, 22], involving different unknown distribution and fragmentation functions. A global analysis of beam and target SSA may be required to separate different contributions. Drell-Yan process with longitudinally and transversely polarized protons scattering on longitudinally polarized protons will also provide additional information on these distribution functions [23].

In conclusion CLAS target SSAs were measured using the $5.7 \mathrm{GeV}$ data from the CLAS experiment using a polarized $\mathrm{NH}_{3}$ target. The twist-2 distribution function $h_{1 L}^{\perp}$ was extracted for the first time using the $\pi^{+}$target SSA. No evidence has been found with CLAS for the large unfavored Collins fragmentation indicated by HERMES [2].

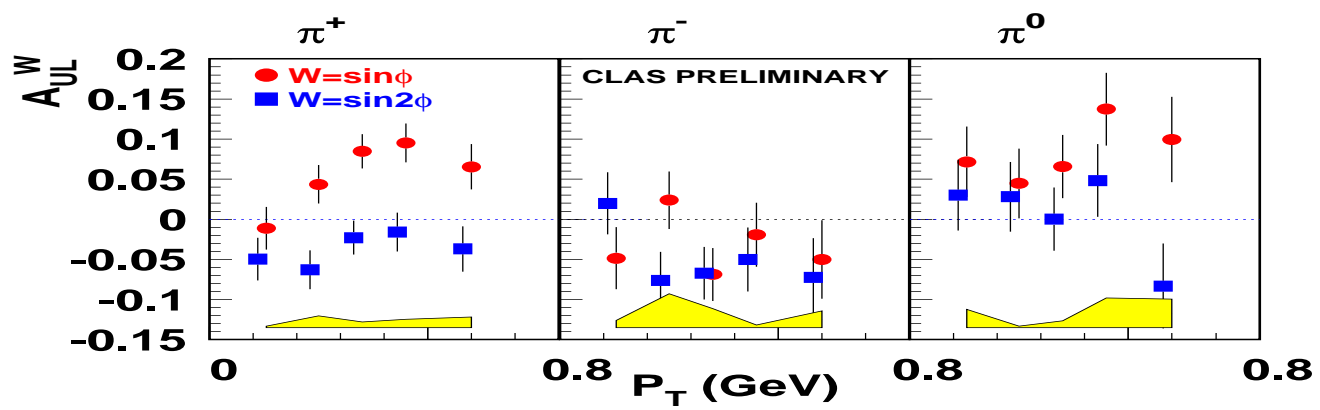

FIGURE 4. The $A_{U L}$ SSA dependence on $P_{\perp}$. The band represents the systematic uncertainty from fit in the measurement of the $\sin 2 \phi$ moment.

\section{REFERENCES}

1. HERMES collaboration (A. Airapetyan et al.), Phys. Rev. Lett. 84, 4047 (2000); Phys. Rev. D64, 097101 (2001); hep-ex/0505042

2. HERMES collaboration (A. Airapetyan et al.), Phys. Rev .Lett. 94, 012002 (2005).

3. CLAS Collaboration (H. Avakian et al.) Phys.Rev. D69, 112004 (2004).

4. D. Sivers, Phys.Rev. D43, 261 (1991).

5. M. Anselmino and F. Murgia, Phys. Lett. B 442, 470 (1998).

6. S. Brodsky et al., Nucl. Phys. B 642, 344 (2002).

7. J. Collins, Phys. Lett. B 536, 43 (2002).

8. X. Ji, F. Yuan, Phys. Lett. B 543, 66 (2002); Nucl. Phys. B 656, 165 (2003).

9. J. Collins, Nucl. Phys. B396, 161 (1993).

10. P.J. Mulders and R.D. Tangerman, Nucl. Phys. B 461, 197 (1996).

11. R. Seidl, this conference.

12. A. Efremov Annalen Phys. 13, 651 (2004)

13. A.M. Kotzinian and P.J. Mulders, Phys. Rev. D54 1229 (1996).

14. J. Ralston and D. Soper, Nucl. Phys. B152, 109 (1979)

15. A. Kotzinian, Nucl. Phys. B 441, 234 (1995). B461, 197 (1996).

16. R.D. Tangerman and P.J. Mulders, Phys.Rev. D51 3357 (1995).

17. B. Mecking et al., Nucl. Inst. \& Meth. 503, 513 (2003).

18. A. V. Efremov et al., Phys. Rev. D 67 (2003) 114014 hep-ph/0412420

19. X. Ji, J-P. Ma and F. Yuan, Phys. Lett. B 597, 299 (2004); Nucl. Phys. B 652, 383 (2003).

20. D. Boer, P. J. Mulders and F. Pijlman, Nucl. Phys. B 667, 201 (2003).

21. A. Bacchetta, P. J. Mulders, and F. Pijlman, Phys. Lett. B595, 309 (2004).

22. K. Goeke, A. Metz, M. Schlegel, hep-ph/0504130

23. R.L. Jaffe and X. Ji, Nucl.Phys. B375 (1992) 527. 